\title{
Real-Time Observation of Stacking Faults in Gold Shock Compressed to 150 GPa
}

\author{
Surinder M. Sharma, ${ }^{1}$ Stefan J. Turneaure, ${ }^{1}$ J. M. Winey, ${ }^{1}$ P. A. Rigg, ${ }^{2}$ N. Sinclair, ${ }^{2}$ \\ Xiaoming Wang, ${ }^{2}$ Y. Toyoda, ${ }^{1}$ and Y. M. Gupta $\odot^{1,3,}$ \\ ${ }^{1}$ Institute for Shock Physics, Washington State University, Pullman, Washington 99164, USA \\ ${ }^{2}$ Dynamic Compression Sector, Institute for Shock Physics, Washington State University, \\ Argonne, Illinois 60439, USA \\ ${ }^{3}$ Department of Physics and Astronomy, Washington State University, Pullman, Washington 99164, USA
}

(Received 23 May 2019; revised manuscript received 15 November 2019; published 15 January 2020)

A microscopic-level understanding of the high-pressure states achieved under shock compression, including comparisons with static compression, is a long-standing and important scientific challenge. Unlike hydrostatic compression, uniaxial strains inherent to shock compression result in plastic deformation and abundant lattice defects. At high pressures (>50 GPa), the role of shock-induced deformation and defects remains an open question. Because of the nanosecond time scales in shock experiments, real-time in situ observations of shock-induced lattice defects have been challenging. Here, we present synchrotron x-ray diffraction measurements on laser-shock-compressed gold that provide the first unambiguous in situ measurements of stacking faults (SFs), likely formed by partial dislocations, during shock compression. SF abundance increases monotonically with shock compression up to $150 \mathrm{GPa}$, where SFs comprise almost every 6th atomic layer. Our results show that SFs play an important role in the plastic deformation of face-centered-cubic metals shocked to high stresses, providing a quantitative benchmark for future theoretical developments.

DOI: $10.1103 /$ PhysRevX.10.011010

\section{INTRODUCTION}

Shock-compression experiments provide an excellent approach to achieve and examine condensed-matter states at high pressures and high temperatures. The combination of shock-wave experiments, static pressure experiments, and advances in theory and computations has been valuable in the development of equations of state (EOS) at extreme conditions $[1,2]$ and in providing insights into structural $[3,4]$ and chemical $[5,6]$ changes in condensed systems.

In addition to accessing extreme thermodynamic states, shock-wave experiments are ideally suited to examine condensed-matter changes in real time (picosecondnanosecond timescales). Although the majority of past studies have involved continuum measurements (e.g., impedance matching and wave profiles) $[1-4,7,8]$, recent experimental advances are providing real-time microscopic information through in situ, x-ray diffraction (XRD) measurements, including new insights into phenomena

\footnotetext{
* Corresponding author. ymgupta@wsu.edu

Published by the American Physical Society under the terms of the Creative Commons Attribution 4.0 International license. Further distribution of this work must maintain attribution to the author(s) and the published article's title, journal citation, and DOI.
}

Subject Areas: Condensed Matter Physics, Materials Science

such as shock-induced structural transformations [9-11] and deformation twinning $[12,13]$.

XRD measurements in shock experiments have the potential to understand the shocked state at the microscopic scale and to permit atomistic level comparisons between the high-pressure states (>50 GPa) achieved in static and shock experiments. To date, such comparisons are made almost exclusively through modeling and simulations [14-16] because high-pressure shock experiments-in contrast to static experiments - have typically provided only continuum or thermodynamic results (pressure, density).

Differences in the thermodynamic states due to the large temperature differences between shock experiments (adiabatic compression) and static experiments (isothermal compression) are well recognized, and accounting for these differences is an important element of high-pressure EOS studies $[1,2,17,18]$. In contrast, the role of differing strains in the high-pressure states achieved under shock and static compression has not received much attention.

Under static high pressure (e.g., diamond anvil cell experiments), materials are subjected to nearly isotropic strains (negligible shear strains). In contrast, materials under shock compression are subjected to uniaxial strain resulting in significant shear strains which can, in turn, generate considerable lattice defects on nanosecond timescales. When interpreting the results of high-pressure shock 
experiments, including comparisons with results from static experiments, the role of shock-induced lattice defects has been largely overlooked.

We address fundamental issues related to strain effects at high pressures using real-time in situ XRD measurements in gold-a representative soft, monatomic cubic metalshock compressed to $150 \mathrm{GPa}$. Gold is chosen for this study because of existing results from shock $[7,19,20]$ and static $[21,22]$ compression experiments and because its equation of state (EOS) [17,23-25] has been established to facilitate its development as a pressure standard. Knowledge of the EOS is important for calculating temperatures in the shocked state.

Dislocation slip - the expected deformation mechanism for shock-compressed gold-has long been inferred as an important mechanism in shocked solids based on the recovery of shock-compressed samples [26], as well as molecular dynamics (MD) simulations of shock-wave propagation [14,15,27-30]. A common finding from phenomenological continuum models and from atomistic simulations is that the dislocation densities estimated during shock compression are several orders of magnitude higher than those found experimentally in shock-recovered samples [29,31]. To resolve and understand these differences, in situ measurements, such as XRD, are needed to examine the role of dislocations during shock compression.

Shock-induced microstructural changes due to dislocation slip are more difficult to discern using real-time in situ measurements, compared to changes due to structural transformations [9-11] and deformation twinning [12,13]. Although dislocations can be examined quantitatively through line broadening using high-resolution, highdynamic-range XRD measurements under static conditions [32], such measurements have not been possible during the short durations (nanosecond timescales) encountered in shock-compression experiments. To date, in situ measurements have been used to infer dislocation activity in shocked solids only indirectly through coarse changes in diffraction patterns, interpreted as lattice rotations $[12,13,33,34]$.

Dislocations in face-centered-cubic (fcc) crystals - such as gold-provide a unique opportunity for in situ observation of dislocation activity during shock compression. The primary slip system in fcc metals is $\{111\}\langle 1 \overline{1} 0\rangle$ and, for this system, it is energetically favorable for a perfect dislocation to dissociate into two Shockley partial dislocations separated by a stacking fault ( $A B C B C A$ stacking of $\{111\}$ planes, instead of $A B C A B C$ stacking) [35]. Thus, perfect dislocation loops comprise narrow (nanometer scale) ribbons of stacking fault (SF), bounded by two concentric partial dislocation loops. In contrast, a partial dislocation loop that lacks a trailing partial leaves behind a SF over the entire loop area [27,36]. Both postmortem examination of shocked fcc solids $[26,36]$ and results from MD simulations $[14,15,27-30]$ suggest that SFs play a significant role in plastic deformation during shock compression of fcc crystals.
The presence of SFs in fcc crystals results in observable changes to their XRD patterns due to SF-related changes in the relative coordinates of neighboring atoms and, hence, the XRD structure factors. In particular, SFs cause both broadening and shifting of XRD peaks, whereas twin faults ( $A B C B A C$ stacking) produce only broadening [37]. The peak shifts due to SFs are $h k l$ dependent, with some peaks — such as $\{200\}$ — shifting to lower scattering angle and other peaks shifting to higher scattering angle [37]. In contrast, lattice strain due to compression of crystalline materials without SFs results in all diffraction peaks shifting to a higher scattering angle, as described by Bragg's law [37]. The peak shifts caused by SFs provide a unique XRD feature that was first observed under ambient conditions using XRD in cold-worked fcc 70-30 $\alpha$ brass [38].

Here, we report on the unambiguous observation of significant stacking-fault abundance in the shockcompressed state-the first such observation at high pressures-using in situ XRD measurements during laser-shock compression of gold. Our results show that analysis of the XRD lines using Bragg's law [37] is inadequate for determining volume compression in shocked gold because results from different $\{h k l\}$ peaks are not consistent. Instead, we demonstrate that an analysis approach incorporating stacking faults is required to determine the volume compression.

\section{IN SITU X-RAY DIFFRACTION AND CONTINUUM MEASUREMENTS}

The experiments were performed at the laser-shock experimental station [39] of the Dynamic Compression Sector located at the Advanced Photon Source, using the configuration shown in Fig. 1. A 100-J laser with 5-ns or 10-ns duration was used to ablate an aluminized Kapton film, resulting in a shock wave in the Kapton which then propagated into $\sim 8-\mu \mathrm{m}$-thick gold foil. Four different laser pulse shapes and energies are used to generate flattop shocked states in the $\mathrm{Au}$ samples with nominal stresses near 40, 65, 120, and $150 \mathrm{GPa}$. Further details about the laser-shock experimental capabilities at the Dynamic Compression Sector can be seen in Ref. [39].

Velocity interferometry [40] is used to record the particle velocity histories at the gold/LiF window interfaces at a location centered on the 500- $\mu \mathrm{m}$ laser-drive spot. Figure 1 shows a typical gold/LiF particle velocity history which has a constant particle velocity for several nanoseconds behind the shock wave indicating a constant stress state. A total of 13 experiments are performed, including several experiments at each nominal shock stress to establish reproducibility of the continuum and XRD results. Results for each experiment are listed in Table I. Gold/LiF particle velocity histories for each experiment are shown in Supplemental Material (SM) Fig. S1 [41]. 


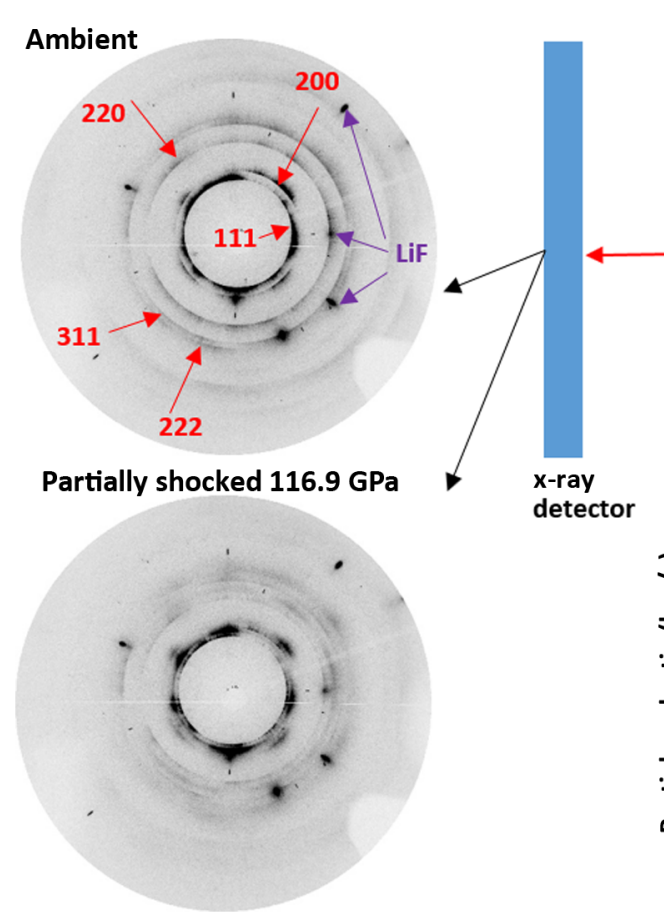

Velocity
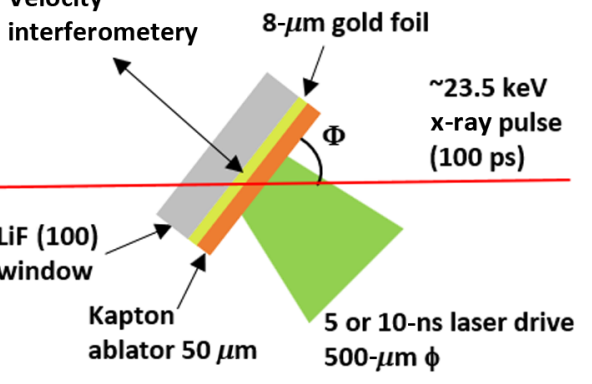

Gold/LiF (100) interface velocity history

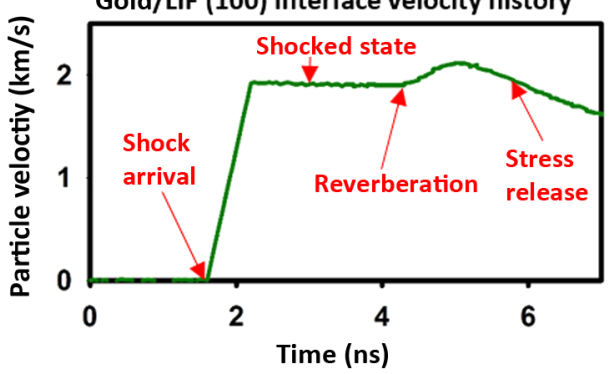

FIG. 1. Schematic diagram for in situ nanosecond x-ray diffraction measurements in shock-compressed gold and representative results. A 351-nm laser pulse is focused on an aluminized Kapton ablator generating a shock wave which then propagates through the $\mathrm{Au}$ sample. XRD patterns corresponding to the ambient Au sample and to the Au sample partially shocked (35\% of Au unshocked during XRD measurement) to $116.9 \mathrm{GPa}$ are shown on the left. The diffraction rings are from the Au and the localized spots are from the single crystal LiF window; a few of the LiF spots are identified in the ambient XRD pattern. The LiF spots are easily identified as they are the same in both XRD patterns because the shock wave had not yet entered the LiF window during the XRD measurement in the laser shock experiments. For the XRD pattern from the partially shocked gold, the diffraction signal from the unshocked portion of the gold is clearly visible in azimuthally integrated 1D line profiles as shown in the Supplemental Material. Laser interferometry is used to measure the gold/LiF window interface velocity from which the shock stress is calculated. Laser drive temporal pulse shapes result in states of constant stress behind the shock wave, as verified by the measured particle velocity histories.

TABLE I. Results for Au laser shock experiments.

\begin{tabular}{|c|c|c|c|c|c|c|}
\hline $\begin{array}{l}\text { Shot } \\
\text { number }\end{array}$ & $\begin{array}{l}\text { Longitudinal } \\
\text { stress }(\mathrm{GPa})\end{array}$ & $\begin{array}{l}\text { Percentage of gold } \\
\text { unshocked during } \\
\text { XRD measurement }\end{array}$ & $\begin{array}{l}\text { Lattice parameter } \\
a \text { determined using } \\
(111) \text { peak }(\AA)^{\mathrm{a}}\end{array}$ & $\begin{array}{l}\text { Lattice parameter } \\
a \text { determined using } \\
(200) \text { peak }(\AA)^{\mathrm{a}}\end{array}$ & $\begin{array}{l}\text { Lattice parameter } \\
a \text { determined using } \\
\text { stacking-fault } \\
\text { analysis }(\AA)^{\mathrm{a}}\end{array}$ & $\begin{array}{l}\text { Stacking-fault } \\
\text { probability }(\alpha)^{\mathrm{b}}\end{array}$ \\
\hline $18 \mathrm{C} 156$ & 0.0 (pre-shot) & 100.0 & 4.078 & 4.078 & 4.078 & 0 \\
\hline $18 C 281$ & $39.1 \pm 0.9$ & 21.0 & 3.867 & 3.877 & 3.867 & 0.07 \\
\hline $18 \mathrm{C} 282$ & $42.3 \pm 0.9$ & 30.0 & 3.862 & 3.875 & 3.862 & 0.09 \\
\hline $18 \mathrm{C} 280$ & $43.5 \pm 0.6$ & 17.2 & 3.855 & 3.870 & 3.855 & 0.09 \\
\hline $18 C 157$ & $63.9 \pm 0.8$ & 5.0 & 3.805 & 3.840 & 3.805 & 0.12 \\
\hline $18 \mathrm{C} 156$ & $65.3 \pm 1.0$ & 25.0 & 3.810 & 3.845 & 3.808 & 0.12 \\
\hline $18 \mathrm{C} 158$ & $66.2 \pm 0.8$ & 6.0 & 3.803 & 3.838 & 3.803 & 0.123 \\
\hline $18 \mathrm{C} 233$ & $116.9 \pm 1.3$ & 35.0 & 3.733 & 3.775 & 3.732 & 0.14 \\
\hline $18 \mathrm{C} 160$ & $120.3 \pm 1.7$ & 18.0 & 3.708 & 3.750 & 3.708 & 0.143 \\
\hline $18 C 159$ & $123.7 \pm 1.8$ & 5.5 & 3.698 & 3.740 & 3.700 & 0.145 \\
\hline $18 \mathrm{C} 176$ & $127.1 \pm 1.3$ & 4.0 & 3.695 & 3.725 & 3.700 & 0.143 \\
\hline $18 \mathrm{C} 177$ & $146.1 \pm 1.5$ & 20.0 & 3.660 & 3.705 & 3.662 & 0.15 \\
\hline $18 \mathrm{C} 178$ & $148.6 \pm 1.5$ & 17.5 & 3.662 & 3.700 & 3.662 & 0.15 \\
\hline $18 \mathrm{C} 236$ & $149.9 \pm 1.5$ & 7.0 & 3.655 & 3.705 & 3.660 & 0.16 \\
\hline
\end{tabular}

${ }^{\mathrm{a}}$ Lattice parameters determined to $\pm 0.005 \AA$.

${ }^{\mathrm{b}}$ Stacking-fault probabilities determined to \pm 0.014 . 
During each experiment, the crystalline structure of the shocked gold sample is probed using an XRD measurement while the high-stress (40-150 GPa) shock wave is propagating through the Au sample. The XRD measurement is recorded before the shock wave has reflected from the $\mathrm{LiF}$ window (the reflection results in a partial stress release) and before the release wave from the ablator enters the Au sample. Thus, the XRD measurements correspond to diffraction from material in two different states: ambient (unshocked) and the peak shocked state. The XRD measurements correspond to a single snapshot obtained using a $\sim 100$-ps duration $\mathrm{x}$-ray pulse with a bandwidth of a few percent and maximum x-ray flux at $\sim 23.5 \mathrm{keV}$ (see SM Fig. S2).

Powder XRD patterns are recorded on an area detector, capturing the first five complete diffraction rings, which are converted to one-dimensional line profiles of intensity versus scattering angle $(2 \theta)$ by integrating around the rings. Because the measured XRD patterns are composed of diffraction from both shocked and unshocked gold, the appropriate fraction of the ambient XRD pattern (measured from the Au sample prior to shock compression) for each experiment is subtracted to obtain the XRD line profile arising from the shocked gold, as described in the SM (see SM Fig. S3). Representative XRD line profiles for ambient

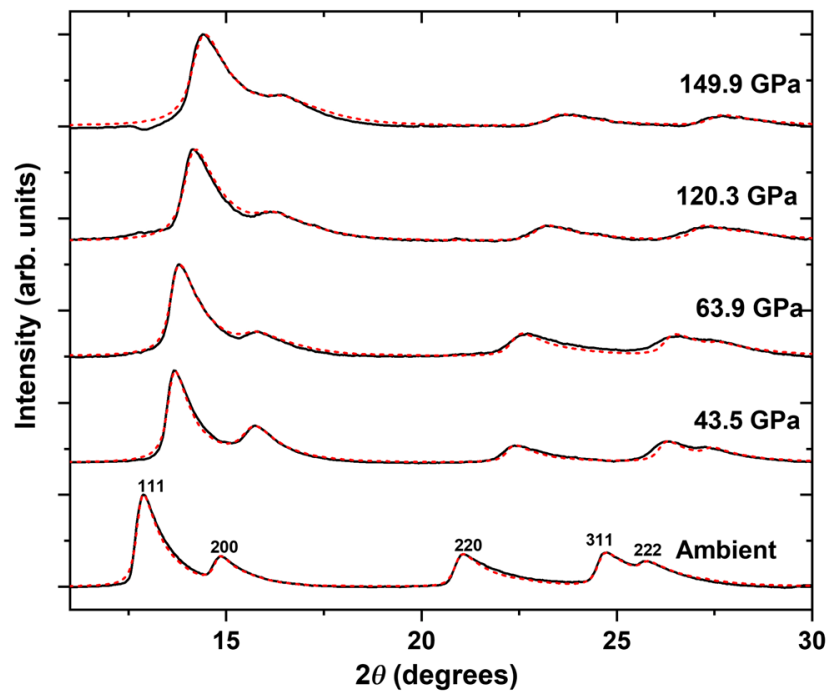

FIG. 2. Shocked gold XRD results. Representative measured and simulated $\mathrm{X}$-ray diffraction line profiles for gold at ambient conditions and for four different shock stresses. Line profiles measured in the shocked states have both the background and the ambient portion of the XRD line profile subtracted. Simulated line profiles (red dashed lines) for shock-compressed Au use lattice parameter $(a)$ and stacking-fault probability $(\alpha)$ values that give the best match to the measured line profiles. The simulation of the ambient line profile uses the ambient $\mathrm{Au}$ lattice constant $a=4.0782 \AA$ and $\alpha=0$. The simulated line profiles are convoluted with a Lorentzian function whose width increases linearly with increasing shock stress (see SM [41]). gold and for gold shocked to the four nominal peak stresses are shown in Fig. 2. More experimental details are given in the SM [41].

\section{ANALYSIS APPROACH FOR STACKING-FAULT DETERMINATION}

Simulated diffraction profiles for ambient $\mathrm{Au}$ and for shocked Au are computed by incorporating the experimental parameters, such as sample thickness, angle of incidence $\Phi$ of the x-ray beam with respect to the sample plane, the spectral flux of $x$ rays incident on the sample, $x$-ray absorption, and instrumental broadening. The relative intensities of different simulated $\{h k l\}$ peaks are allowed to vary when fitting to the overall measured line profiles to account for texture effects. More details regarding the simulations are provided in the SM [41]. The presence of stacking faults affects the shapes and positions of the various $\{h k l\}$ peaks. Below, we describe how these SF effects are incorporated in the diffraction simulations.

The basic formalism for evaluating the effect of SFs and twin faults on x-ray diffraction patterns for fcc materials was given by Warren [37]. This formalism was extended by Velterop et al. [45], to allow accurate determination of stacking-fault $(\alpha)$ and twin-fault $(\beta)$ probabilities, even when $\alpha$ and $\beta$ become large. Here, $1 / \alpha$ and $1 / \beta$ indicate the average number of $\{111\}$ planes between stacking faults and twin faults, respectively.

For shocked $\mathrm{Au}$, the effect of stacking faults is incorporated in our XRD simulations using the formulation of Ref. [45] to independently calculate line profiles for each $\{h k l\}$ diffraction peak. The formalism considers stacking faults on (111) planes with a crystal consisting of $N_{p}$ (111) layers. $N_{p}$ is fixed at 10000 [41]; lower $N_{p}$ values resulted in increased widths of all the peaks due to size broadening. Because the formalism incorporates nonlinear terms of stacking-fault probability $(\alpha)$ and twinning fault probability $(\beta)$, its applicability extends to large values of $\alpha$ and $\beta$. For stacking faults on (111) planes, some $(h k l)$ peaks from a given $\{h k l\}$ family are shifted due to stacking faults (affected components) while other $(h k l)$ peaks are not shifted (unaffected components) [37,45]. For a given $\{h k l\}$ family, the total line profile is the superposed sum of the affected and unaffected components. The affected components have $\left|L_{0}\right| \equiv|h+k+l|=3 J \pm 1$ ( $J$ an integer) and the unaffected components have $\left|L_{0}\right|=3 J$ ( $J$ an integer). The relative intensities of the affected and unaffected components for a given $\{h k l\}$ include appropriate multiplicity factors, as described by Velterop et al. [45]. The relative intensities of the affected and unaffected components also vary substantially with increasing $\alpha$ [45]. The lattice parameter $(a)$ and stacking-fault probability $(\alpha)$ in the shocked state are determined by carrying out simulations varying $a$ and $\alpha$ (and maintaining $\beta=0$, as discussed in Sec. IV B) to obtain a good match to the measured diffraction profiles. The simulations also 
incorporate an additional Lorentzian function which is convoluted with the simulated line profiles to account for instrumental broadening as well as broadening due to material heterogeneities (size broadening, strain broadening, twin faults, etc.) other than stacking faults.

\section{RESULTS}

\section{A. Lattice compression from XRD peak shifts}

Lattice plane spacings $d_{h k l}$ and lattice parameter $a$ are typically determined from the diffraction peak scattering angle $2 \theta$ and the x-ray wavelength $\lambda$ using Bragg's law.

$$
\lambda=2 d_{h k l} \sin \theta
$$

As expected, clear shifts of the diffraction peaks to a higher scattering angle are observed with increasing stress, due to increased compression (see Fig. 2). For isotropic compression of cubic metals, the lattice parameter $a$ and the volume $V / V_{0}=\left(a / a_{0}\right)^{3}$ should be independent of which ( $h k l$ ) diffraction peak is examined; simulated diffraction patterns confirm this for the ambient gold samples (see Table I). However, for shock-compressed $\mathrm{Au}$, the diffraction simulations show that the lattice parameter $a$ and volume $V / V_{0}=\left(a / a_{0}\right)^{3}$ differ substantially depending on which $(h k l)$ peak is used to determine $a$ (see SM and

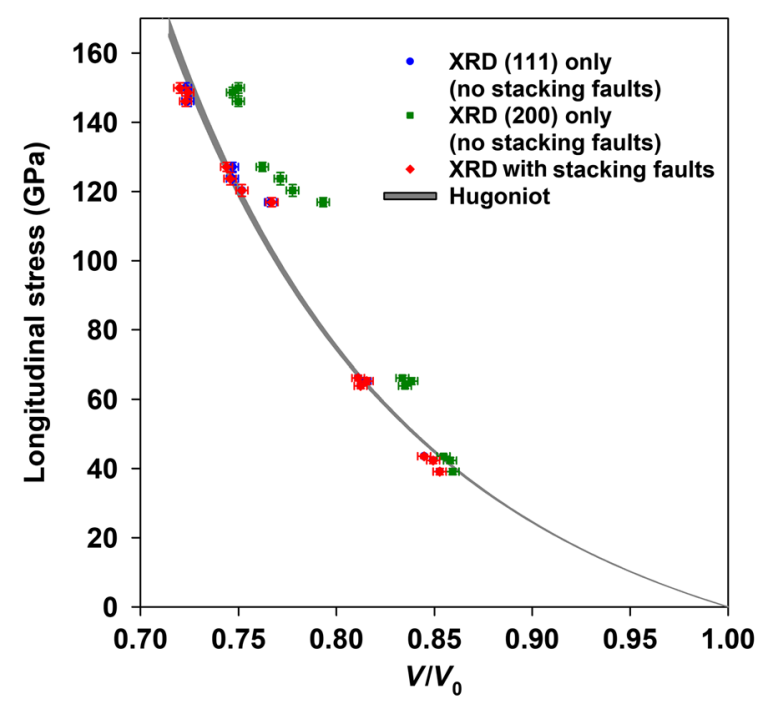

FIG. 3. Stress-volume states of shock-compressed gold. Stresses are calculated from velocity interferometry data. Unit cell volumes are calculated from $a^{3}$, where the lattice constant $a$ is determined directly from a fit to the (111) diffraction peak (blue circles) or the (200) diffraction peak (green squares), without considering the effects of stacking faults. The red diamonds are from a combined fit of the full diffraction profile, comprising all five peaks, that incorporates shifting and broadening of the diffraction peaks due to stacking faults. The gray band is the Hugoniot curve for gold, determined from continuum measurements (Refs. [7,19,20]; see SM [41]); the width of the band indicates the experimental uncertainty.
Fig. S4 [41]). As shown in Fig. 3, volumes determined using the (200) diffraction peaks are significantly larger than volumes expected from the known Au Hugoniot curve [41]. In contrast, volumes determined using the (111) diffraction peaks provide a reasonable match to those determined from the Au Hugoniot [41]. These apparently contradictory results can be reconciled by considering the effects of stacking faults on the various $(h k l)$ diffraction peaks of an fcc material, as presented next.

\section{B. Determination of stacking-fault probability}

As discussed in Sec. III, we simulated the diffraction profiles for each experiment, varying the lattice parameter (a), the fault probabilities ( $\alpha$ and $\beta$ ), and the width of the Lorentzian broadening function to obtain the best match to the measured diffraction profiles. Our simulations show that the simulated profiles have a very weak dependence on $\beta$, which primarily affects broadening (see SM Fig. S5 [41]). Because peak broadening can also arise from other sources, such as strain distributions, nonisotropic compression, or small coherently diffracting domain size, we cannot extract quantitative values for the twin-fault probability from our measurements. However, the stacking-fault probabilities determined by fitting the simulations to the measured line profiles are found to be insensitive to the choice of $\beta$. Therefore, $\beta$ is fixed at zero for all final simulations. The values of stacking-fault probability $\alpha$ determined from our analysis are found to be insensitive to the value of $N_{p}$ used in the analysis as discussed in the SM [41]. We also could not detect any sensitivity of the stacking-fault probabilities due to texture effects [41].

Figure 2 shows representative simulated diffraction profiles (with best-fit $a$ and $\alpha$ values) at each of the four shock-stress levels. The simulated line profile for the ambient gold sample shows excellent agreement with the measured line profile, without the inclusion of faults $(\alpha=\beta=0)$. However, good matches between simulations and measured peak positions and peak widths for the shocked samples are obtained only for nonzero $\alpha$. The sensitivity of the simulations to the value of $\alpha$ is shown in SM Fig. S6 [41], where the simulated diffraction patterns with $\alpha=0$ for $\mathrm{Au}$ shocked to several representative stresses result in a much narrower (200) peak, located at a higher scattering angle, than observed in the measured line profile. The simulated (200) peak width and location match the measured line profile significantly better after incorporating the effect of SFs. Thus, the finite stackingfault probability $\alpha$ has a significant and quantifiable effect on the diffraction line profiles of shocked Au.

Because of the heterogeneous nature of inelastic deformation, increased widths of diffraction peaks are expected for materials shock compressed beyond their elastic limit $[33,46]$. These shock-induced microstructural heterogeneities lead to smaller coherently diffracting domains. This feature is incorporated in the simulated 
profiles shown in Fig. 2 by convoluting the computed profiles with Lorentzian line shapes having widths that increase linearly with shock stress between the Lorentzian width needed to match the ambient line profile and that needed to match the highest stress line profile $(\sim 2.5$ times increase in width, compared to ambient; see SM [41]). The variations in the line shapes as a function of diffraction angle are represented by dotted curves in Fig. S7. We note that smaller coherently diffracting domain volumes and associated line broadening is consistent with the increase in stacking-fault density. At the highest stress $(\sim 150 \mathrm{GPa})$, if all additional Lorentzian broadening due to material properties is attributed to size broadening, the Scherrer equation gives a coherently diffracting domain size of $\sim 8 \mathrm{~nm}$. However, this represents a lower bound because other heterogeneities such as lattice strain distributions and twins can also contribute to this broadening. Although we cannot quantitatively determine the contributions of the various heterogeneities (other than stacking faults) to this additional Lorentzian broadening, we find that the SF probability $(\alpha)$ determined from the relative (200) peak shift is insensitive to whether this additional broadening is included or not. The additional broadening simply improves the match between the measured and simulated line profiles.

The presence of SFs provides an explanation for the different volumes determined from the (111) diffraction peak and from the (200) diffraction peak (Fig. 3). As discussed in Sec. III, SFs on the (111) plane cause some of the $\{111\}$ component peaks to shift to higher scattering angles (affected peaks), whereas other $\{111\}$ component peaks do not shift (unaffected peaks). However, because the intensity of affected components rapidly decreases with increasing $\alpha$ [45], the overall shift of the integrated $\{111\}$ diffraction peak due to SFs is small. In contrast, all components of the $\{200\}$ peak are affected, shifting to a lower scattering angle for finite $\alpha$. As a result, volumes determined using the $\{200\}$ peak, without considering the effects of SFs, are larger than those determined using the $\{111\}$ peak (Fig. 3). However, when the effects of SFs are incorporated, the best-fit simulated diffraction profiles (Fig. 2) provide volumes that are consistent across all five of the fitted diffraction peaks. The volumes from these simulations, shown as the red symbols in Fig. 3, provide a good overall match to the volumes determined from the Au Hugoniot curve [41] (see also SM Fig. S8).

Figure 4 shows how the stacking-fault probability $\alpha$ for shocked gold, deduced from the XRD data, varies with volume compression, $\eta=\left(1-V / V_{0}\right)$. Longitudinal stresses and calculated temperatures corresponding to the volume compressions [41] are also shown for completeness. Overall, the SF probability increases monotonically with compression: $\alpha$ increases rapidly up to $\eta=0.18(\sim 60 \mathrm{GPa}$ stress $)$, followed by a smaller increase at larger compressions. The SF probability reaches $\sim 0.16$

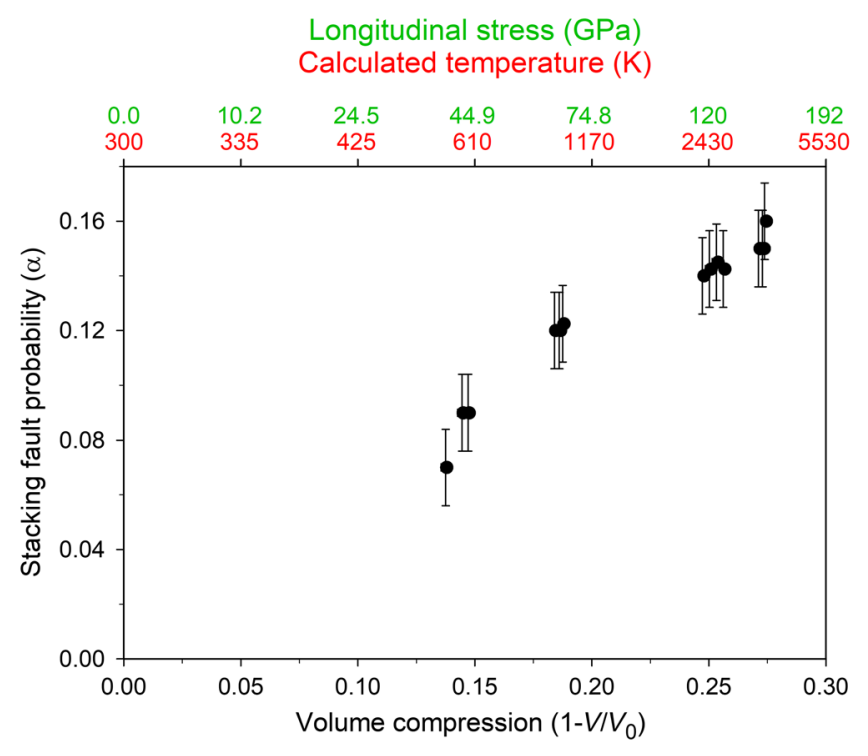

FIG. 4. Stacking-fault probability versus volume compression. Stacking-fault probability $\alpha$ increases monotonically with volume compression. Corresponding longitudinal stresses and calculated temperatures are shown at the top. The calculated temperatures are determined using the equation of state from Ref. [17]. At the lowest stresses ( 40 GPa), and as discussed in the SM [41], potential contributions of strength effects on the stacking-fault probabilities cannot be ruled out.

near $\eta=0.28(\sim 150$ GPa stress). Statistically, $\alpha=0.16$ implies that approximately every sixth atomic layer is a stacking fault.

We have also considered the possible effects of strength on our measured XRD profiles. As discussed in the SM [41], our analysis suggests that strength effects are negligible at high stresses. However, at the lowest stresses examined here ( $\sim 40 \mathrm{GPa})$, we are unable to entirely rule out possible contributions to the XRD profiles due to strength effects.

\section{DISCUSSION}

Based on early in situ XRD measurements, evidence for SFs in shock-compressed aluminum was claimed [47]. However, other early XRD studies on shocked $\mathrm{Al}$ reported no such evidence $[48,49]$. Moreover, recent high-resolution in situ XRD measurements [50] on shock-compressed Al single crystals show that all of the observed peaks (irrespective of $h \mathrm{kl}$ ) give the same lattice constant using Bragg's law; this finding does not support the earlier claim of a measurable density of SFs in shocked Al. Although MD simulations have predicted stacking-fault formation in $\mathrm{Cu}$ single crystals shocked along the [100] direction [29], in situ Laue XRD measurements on shocked [100] $\mathrm{Cu}$ single crystals are used to conclude that the observed diffraction spot changes do not indicate stacking-fault formation [34].

The results presented here for shocked gold are the first in situ observations of the generation of partial dislocations 
and SFs during shock-wave compression to high stresses. These results provide significant insight into the mechanisms governing plastic deformation in shocked fcc materials and constitute an important step in addressing long-standing questions regarding the role of dislocations in the very rapid plastic deformation encountered during shock compression.

Stacking-fault formation during shock compression along specific crystalline directions (mostly [100]) was previously examined in fcc metals, other than gold, using MD simulations $[14,29,30]$. In those studies, $a_{0} /\langle l\rangle$ was plotted versus $u_{p} / c_{0}$, where $a_{0}$ is the fcc lattice parameter, $\langle l\rangle$ is the average spacing between SFs, $u_{p}$ is the particle velocity behind the shock wave, and $c_{0}$ is the longitudinal sound speed. Noting that the stacking-fault probability $\alpha$ is proportional to $a_{0} /\langle l\rangle$ (referred to as a measure of plasticity in Refs. $[14,29,30])$ and $u_{p} / c_{0}$ is related to the longitudinal shock stress, we find that the largest SF probability in Fig. 4 ( $\alpha \sim 0.16$, corresponding to $a_{0} /\langle l\rangle \sim 0.32$ ) is roughly consistent with the values reported from MD simulations for other fcc solids $[14,29,30]$. Because stacking-fault energies are highly material specific, and no MD simulations on shock propagation in Au have been carried out, a more quantitative comparison is not currently possible. However, Fig. S9 shows that the variation in SF abundance observed in our work has qualitative features similar to those predicted in MD simulations [14]. Moreover, the SF probabilities shown in Fig. 4 are several orders of magnitude larger than those found in samples recovered after shock-compression experiments [29], suggesting that annihilation of SFs likely occurs during release from the shocked state. XRD measurements during release are needed to definitively address the question of SF annihilation.

We note that, in MD simulations on fcc single crystals, SF generation depends on the propagation direction of the shock wave [14,15,27-30]. In addition, MD simulations have shown that SF generation also takes place at the grain boundaries in polycrystalline samples [30,51]. Our XRD studies probe $a \sim 10^{5} \mu \mathrm{m}^{3}$ volume in the polycrystalline gold. Therefore, our results represent an average over different crystalline directions and could possibly include contributions from SFs generated at the grain boundaries.

In the analysis presented above, multiple measured powder XRD peaks are required to properly incorporate the effects of stacking faults and to determine volumes that are consistent for all the measured peaks. Thus, a significant finding from our work is that volume determinations for shocked fcc metals - and perhaps for other materials, as well-using a single measured powder XRD peak can be in error if the position of the chosen $(h k l)$ peak (such as 200 for shocked $\mathrm{Au}$ ) is sensitive to defects. Therefore, multiple measured peaks should be examined, when possible.

For the compression of gold under quasihydrostatic conditions, the observed diffraction pattern indicates locally hydrostatic compression and no signature of SFs, even up to terapascal pressures [52]. Therefore, the generation of SFs in shock-compressed gold is related to the uniaxial strain state (and associated shear strains) inherent to plane shock-wave compression. This finding demonstrates that comparing high-pressure XRD results from shock compression and static compression needs to incorporate strain effects.

The observation of a large SF probability in shocked gold has potential significance regarding the use of goldand perhaps other metals, as well-as a pressure standard in diamond anvil cell experiments. Because of the low strength of gold at ambient conditions, previous determinations of the gold-pressure standard [17,23-25] assumed that the high stress in shocked gold is characterized by an isotropic stress state (i.e., no strength). Because shockinduced defect generation-such as the large measured SF probabilities reported here- has been previously associated with strain hardening or an increase in strength in shocked metals [53], the previous assumption of an isotropic stress state for shocked gold should be examined carefully.

The large SF probabilities suggest that a significant fraction of the work done by plastic deformation is not dissipated as heat, but instead is stored in the configurational energy of the defect structures. Such energy storage, which was ignored in previous gold-pressure standard determinations [17,23-25], could potentially have a measurable effect on isothermal compression curves determined from the measured gold Hugoniot states. Therefore, theoretical calculations are needed to quantitatively estimate energy storage by defects in shocked gold and to examine possible consequences regarding the use of gold as a pressure standard.

It was previously argued [18] that the high temperatures encountered at high shock stresses $(\sim 3600 \mathrm{~K}$ for gold at $150 \mathrm{GPa}$ [17]) would lead to a rapid annealing of defects, resulting in reduced strength at high stresses and a reduced effect of defects on pressure standard determinations. However, the large and increasing SF probabilities reported here suggest that significant annealing does not take place on the nanosecond timescales of our experiments.

\section{CONCLUDING REMARKS}

In conclusion, the present investigation using real-time in situ XRD measurements to examine gold shock compressed to high stresses provides detailed microscopic information about the shocked state that cannot be obtained using other currently available experimental methods. The results presented here provide insight into the role of strains in understanding the high-pressure $(\sim 100 \mathrm{GPa})$ states achieved under shock compression (uniaxial strain) and under static compression (nearly isotropic strains). Lattice defects resulting from plastic deformation during shock compression are shown to play an important role at high pressures, demonstrating that density and temperature alone do not fully characterize the high-pressure shocked 
state, even for a soft monatomic cubic metal, such as gold. The abundance of lattice defects in shock-compressed gold suggests that shock-induced microstructure likely plays an important role in other shocked solids, as well. Shear strains (and resulting defects) under shock compression have long been conjectured [3,5,54] as likely being responsible for the rapid kinetics associated with shockinduced phase transitions and chemical reactions. The present work constitutes an important experimental step in gaining insight into this important issue. Our findings also demonstrate the need to incorporate shock-induced lattice defects in theoretical calculations for shocked solids. In particular, our quantitative analysis to determine SF probability in the shock-compressed state provides an important benchmark against which future theoretical developments can be tested.

\section{ACKNOWLEDGMENTS}

Yuelin Li, Adam Schuman, Nicholas Weir, and Jun Zhang at the Dynamic Compression Sector (Advanced Photon Source, Argonne National Laboratory) are gratefully acknowledged for their expert assistance with the experiments. The analysis to estimate the effects of strength on the measured XRD profiles-mentioned here and described in the SM [41] — was carried out at the suggestion of anonymous referees. This publication is based upon work supported by the U.S. Department of Energy (DOE), National Nuclear Security Administration (NNSA) under Award No. DE-NA0002007. This publication is also based upon work performed at the Dynamic Compression Sector, which is operated by Washington State University under DOE/NNSA Award No. DE-NA0002442. This research used resources of the Advanced Photon Source, a DOE Office of Science User Facility operated for the DOE Office of Science by Argonne National Laboratory under Contract No. DE-AC02-06CH11357.

[1] W. J. Nellis, J. A. Moriarty, A. C. Mitchell, M. Ross, R. G. Dandrea, N. W. Ashcroft, N. C. Holmes, and G. R. Gathers, Metals Physics at Ultrahigh Pressure: Aluminum, Copper, and Lead as Prototypes, Phys. Rev. Lett. 60, 1414 (1988).

[2] M. D. Knudson and M. P. Desjarlais, Shock Compression of Quartz to 1.6 TPa: Redefining a Pressure Standard, Phys. Rev. Lett. 103, 225501 (2009).

[3] G. E. Duvall and R. A. Graham, Phase Transitions under Shock-Wave Loading, Rev. Mod. Phys. 49, 523 (1977).

[4] M. D. Knudson, M. P. Desjarlais, and D. H. Dolan, ShockWave Exploration of the High-Pressure Phases of Carbon, Science 322, 1822 (2008).

[5] J. J. Gilman, Mechanochemistry, Science 274, 65 (1996).

[6] E. J. Reed, M. R. Manaa, L. E. Fried, K. R. Glaesemann, and J. D. Joannopoulos, A Transient Semimetallic Layer in Detonating Nitromethane, Nat. Phys. 4, 72 (2008).
[7] LASL Shock Hugoniot Data, edited by S. P. Marsh (University of California Press, Los Angeles, 1980), p. 81.

[8] D. Choudhuri and Y. M. Gupta, Shock Compression of Aluminum Single Crystals to 70 GPa: Role of Crystalline Anisotropy, J. Appl. Phys. 114, 153504 (2013).

[9] T. d'Almeida and Y. M. Gupta, Real-Time X-Ray Diffraction Measurements of the Phase Transition in $\mathrm{KCl}$ Shocked along [100], Phys. Rev. Lett. 85, 330 (2000).

[10] D. H. Kalantar, J. F. Belak, G. W. Collins, J. D. Colvin, H. M. Davies, J. H. Eggert, T. C. Germann, J. Hawreliak, B. L. Holian, K. Kadau, P. S. Lomdahl, H. E. Lorenzana, M. A. Meyers, K. Rosolankova, M. S. Schneider, J. Sheppard, J. S. Stolken, and J. S. Wark, Direct Observation of the $\alpha-\varepsilon$ Transition in Shock-Compressed Iron via Nanosecond X-Ray Diffraction, Phys. Rev. Lett. 95, 075502 (2005).

[11] S. J. Turneaure, N. Sinclair, and Y. M. Gupta, Real-Time Examination of Atomistic Mechanisms during ShockInduced Structural Transformation in Silicon, Phys. Rev. Lett. 117, 045502 (2016).

[12] C. E. Wehrenberg, D. McGonegle, C. Bolme, A. Higginbotham, A. Lazicki, H. J. Lee, B. Nagler, H.-S. Park, B. A. Remington, R. E. Rudd, M. Sliwa, M. Suggit, D. Swift, F. Tavella, L. Zepeda-Ruiz, and J. S. Wark, in situ X-Ray Diffraction Measurement of Shock-Wave-Driven Twinning and Lattice Dynamics, Nature (London) 550, 496 (2017).

[13] S. J. Turneaure, P. Renganathan, J. M. Winey, and Y. M. Gupta, Twinning and Dislocation Evolution during Shock Compression and Release of Single Crystals: Real-Time X-Ray Diffraction, Phys. Rev. Lett. 120, 265503 (2018).

[14] B. L. Holian and P. S. Lomdahl, Plasticity Induced by Shock Waves in Nonequilibrium Molecular-Dynamics Simulations, Science 280, 2085 (1998).

[15] T. C. Germann, B. L. Holian, P. S. Lomdahl, and R. Ravelo, Orientation Dependence in Molecular Dynamics Simulations of Shocked Single Crystals, Phys. Rev. Lett. 84, 5351 (2000).

[16] V. I. Levitas and R. Ravelo, Virtual Melting as a New Mechanism of Stress Relaxation under High Strain Rate Loading, Proc. Natl. Acad. Sci. U.S.A. 109, 13204 (2012).

[17] C. W. Greeff and M. J. Graf, Lattice Dynamics and the High-Pressure Equation of State of Au, Phys. Rev. B 69 , 054107 (2004).

[18] A. D. Chijioke, W. J. Nellis, and I. F. Silvera, High-Pressure Equations of State of $\mathrm{Al}, \mathrm{Cu}, \mathrm{Ta}$, and W, J. Appl. Phys. 98, 073526 (2005).

[19] L. V. Altshuler, A. A. Bakanova, I. P. Dudoladov, E. A. Dynin, R. F. Trunin, and B.S. Chekin, Shock Adiabatic Curves of Metals. New Data, Statistical Analysis, and General Laws, J. Appl. Mech. Tech. Phys. 22, 145 (1981).

[20] M. Yokoo, N. Kawai, K. G. Nakamura, and K. Kondo, Hugoniot Measurement of Gold at High Pressures of up to 580 GPa, Appl. Phys. Lett. 92, 051901 (2008).

[21] A. Dewaele, P. Loubeyre, and M. Mezouar, Equations of State of Six Metals above 94 GPa, Phys. Rev. B 70, 094112 (2004).

[22] K. Takemura and A. Dewaele, Isothermal Equation of State for Gold with a He-Pressure Medium. Phys. Rev. B 78, 104119 (2008). 
[23] J. C. Jamieson, J. N. Fritz, and M. H. Manghanani, Pressure Measurement at High Temperature in X-Ray Diffraction Studies: Gold as a Primary Standard, in High-Pressure Research in Geophysics, edited by S. Akimoto and M. H. Manghnani (Center for Academic Publications, Tokyo, 1982), pp. 27-48.

[24] S. H. Shim, T. S. Duffy, and T. Kenichi, Equation of State of Gold and Its Application to the Phase Boundaries near $660 \mathrm{~km}$ Depth in Earth's Mantle, Earth Planet. Sci. Lett. 203, 729 (2002).

[25] M. Yokoo, N. Kawai, K. G. Nakamura, K. I. Kondo, Y. Tange, and T. Tsuchiya, Ultrahigh-Pressure Scales for Gold and Platinum at Pressures up to 550 GPa, Phys. Rev. B 80, 104114 (2009).

[26] L. E. Murr, Residual Microstructure-Mechanical Property Relationships in Shock-Loaded Metals and Alloys, in Shock Waves and High-Strain-Rate Phenomena in Metals, edited by M. A. Myers and L. E. Murr (Plenum Press, New York, 1981), pp. 607-673.

[27] T. C. Germann, D. Tanguy, B. L. Holian, P. S. Lomdahl, M. Mareschal, and R. Ravelo, Dislocation Structure behind a Shock Front in fcc Perfect Crystals: Atomistic Simulation Results, Metall. Mater. Trans. A 35, 2609 (2004).

[28] K. Rosolankova, J. S. Wark, E. M. Bringa, and J. Hawreliak, Measuring Stacking Fault Densities in Shock-Compressed FCC Crystals Using In Situ X-Ray Diffraction, J. Phys. Condens. Matter 18, 6749 (2006).

[29] B. Cao, E. M. Bringa, and M. A. Meyers, Shock Compression of Monocrystalline Copper: Atomistic Simulations, Metall. Mater. Trans. A 38, 2681 (2007).

[30] H. N. Jarmakani, E. M. Bringa, P. Erhart, B. A. Remington, Y. M. Wang, N. Q. Vo, and M. A. Meyers, Molecular Dynamics Simulations of Shock Compression of Nickel: From Monocrystals to Nanocrystals, Acta Mater. 56, 5584 (2008).

[31] M. A. Meyers, A Mechanism for Dislocation Generation in Shock-Wave Deformation. Scr. Metall. 12, 21 (1978).

[32] A. Borbely and T. Ungar X-Ray Line Profile Analysis of Plastically Deformed Metals, C.R. Phys. 13, 293 (2012).

[33] S. J. Turneaure, Y. M. Gupta, K. Zimmerman, K. Perkins, C. S. Yoo, and G. Shen, Real-Time Microstructure of Shocked LiF Crystals: Use of Synchrotron X-Rays, J. Appl. Phys. 105, 053520 (2009).

[34] M. J. Suggit, A. Higginbotham, J. A. Hawreliak, G. Mogni, G. Kimminau, P. Dunne, A. J. Comley, N. Park, B. A. Remington, and J.S. Wark, Nanosecond White-Light Laue Diffraction Measurements of Dislocation Microstructure in Shock-Compressed Single-Crystal Copper, Nat. Commun. 3, 1224 (2012).

[35] J. P. Hirth and J. Lothe, Theory of Dislocations (McGrawHill, New York, 1968).

[36] M. A. Meyers, M. S. Schneider, H. Jarmakani, B. Kad, B. A. Remington, D. H. Kalantar, J. Mcnaney, B. Cao, and J. Wark, Deformation Substructures and Their Transitions in Laser Shock-Compressed Copper-Aluminum Alloys, Metall. Mater. Trans. A 39, 304 (2008).

[37] B. E. Warren, X-ray Diffraction (Addison-Wesley, Reading, 1969).

[38] B. E. Warren and E. P. Warekois, Measurements of Stacking Faults in Cold-Worked Alpha Brass, J. Appl. Phys. 24, 951 (1953).
[39] X. Wang, P. Rigg, J. Sethian, N. Sinclair, N. Weir, B. Williams, J. Zhang, J. Hawreliak, Y. Toyoda, Y. M. Gupta, Y. Li, D. Broege, J. Bromage, R. Earley, D. Guy, and J. Zuegel, The Laser Shock Station in the Dynamic Compression Sector, Rev. Sci. Instrum. 90, 053901 (2019).

[40] L. M. Barker and R. E. Hollenbach, Laser Interferometer for Measuring High Velocities of Any Reflecting Surface, J. Appl. Phys. 43, 4669 (1972).

[41] See Supplemental Material at http://link.aps.org/ supplemental/10.1103/PhysRevX.10.011010 for additional details regarding the experiments and analysis. The Supplemental Material contains Refs. [42-44].

[42] P. A. Rigg, M. D. Knudson, R. J. Scharff, and R. S. Hixson, Determining the Refractive Index of Shocked [100] Lithium Fluoride to the Limit of Transmissibility, J. Appl. Phys. 116, 033515 (2014).

[43] A. P. Hammersley, FIT2D: An Introduction and Overview, ESRF Internal Report No. ESRF97-HA02T, 1997.

[44] A. K. Singh, C. Balasingh, H.-K. Mao, R. J. Hemley, and J. Shu, Analysis of Lattice Strains Measured under Nonhydrostatic Pressure, J. Appl. Phys. 83, 7567 (1998).

[45] L. Velterop, R. Delhez, T. H. de Keijser, E. J. Mittemeijer, and D. Reefman, X-Ray Diffraction Analysis of Stacking and Twin Faults in f.c.c. Metals: A Revision and Allowance for Texture and Non-Uniform Fault Probabilities, J. Appl. Crystallogr. 33, 296 (2000).

[46] S. J. Turneaure and Y. M. Gupta, Real-Time Microstructure of Shock-Compressed Single Crystals from X-ray Diffraction Line Profiles, J. Appl. Crystallogr. 44, 574 (2011).

[47] E. B. Zaretsky, X-Ray Diffraction Evidence for the Role of Stacking Faults in Plastic Deformation of Solids under Shock Loading, Shock Waves 2, 113 (1992).

[48] Q. Johnson, A. C. Mitchell, and L. Evans, X-Ray Diffraction Study of Single Crystals Undergoing Shock-Wave Compression, Appl. Phys. Lett. 21, 29 (1972).

[49] L. V. Altshuler, L. A. Egorov, E. V. Nitochkina, and Y. K. Orekin, X-Ray Diffraction Investigation of the Structure of Shock-Compressed Aluminum, Sov. Phys. JETP 54, 359 (1981).

[50] S. J. Turneaure and Y. M. Gupta, Material Strength Determination in the Shock Compressed State Using X-Ray Diffraction Measurements, J. Appl. Phys. 109, 123510 (2011).

[51] V. Yamakov, D. Wolf, S. R. Phillpot, A. K. Mukherjee, and H. Gleiter, Deformation-Mechanism Map for Nanocrystalline Metals by Molecular-Dynamics Simulation, Nat. Mater. 3, 43 (2004).

[52] N. Dubrovinskaia, L. Dubrovinsky, N. A. Solopova, A. Abakumov, S. Turner, M. Hanfland, E. Bykova, M. Bykov, C. Prescher, V. B. Prakapenka, S. Petitgirard, I. Chuvashova, B. Gasharova, Y. L. Mathis, P. Ershov, I. Snigireva, and A. Snigirev, Terapascal Static Pressure Generation with Ultrahigh Yield Strength Nanodiamond, Sci. Adv. 2, e1600341 (2016).

[53] J. J. Gilman, Micromechanics of Flow in Solids (McGrawHill, New York, 1969).

[54] Shock-Compression Chemistry in Materials Synthesis and Processing, National Materials Advisory Board (NMAB) Report No. 414, 1984. 